\title{
Monostatic Indoor Localization: Bounds and Limits
}

\author{
Gregor Dumphart*, Erik Leitinger $^{\dagger}$, Paul Meissner ${ }^{\dagger}$, and Klaus Witrisal ${ }^{\dagger}$ \\ * Communication Technology Laboratory, ETH Zurich, Switzerland, Email: dumphart@nari.ee.ethz.ch \\ $\dagger$ Graz University of Technology, Austria, Email: \{erik.leitinger, paul.meissner, witrisal\}@ tugraz.at
}

\begin{abstract}
Multipath-assisted localization is a promising concept for cooperative agent networks to guarantee robust and accurate positioning in indoor environments with harsh radio channel characteristics. It uses two types of measurements: (i) bistatic measurements between agents and (ii) monostatic (batlike) measurements by the individual agents. This paper presents an in-depth analysis of the position-related information provided by monostatic measurements. The CRLB on the position error is computed and used to analyze the impact of the indoor geometry and to find the limitations of such measurements. A general form of multipath delay gradients, crucial terms in the CRLB relating geometry and channel parameters, is derived for this purpose.
\end{abstract}

\section{INTRODUCTION}

Multipath-assisted indoor localization [1]-[3] is able to robustly achieve a high level of accuracy in indoor environments with harsh multipath conditions. Ultra-wideband (UWB) systems provide the needed separability of multipath components (MPCs). With an a priori known floor plan, these MPCs can be linked to the local geometry and used as (additional) virtual anchors (VAs) [4]. All other, not geometrically modeled propagation effects constitute interference to the exploitable position-related information and are called diffuse multipath (DM) [5]. Our work [2] has shown the significant increase in performance when using prior knowledge of a geometricstochastic channel model.

In [6] we showed that multipath-assisted concepts are applicable to a cooperative setup where agents perform two types of measurements (cf. Figure 1): (i) bistatic measurements between agents and (ii) monostatic (bat-like) measurements. ${ }^{1}$

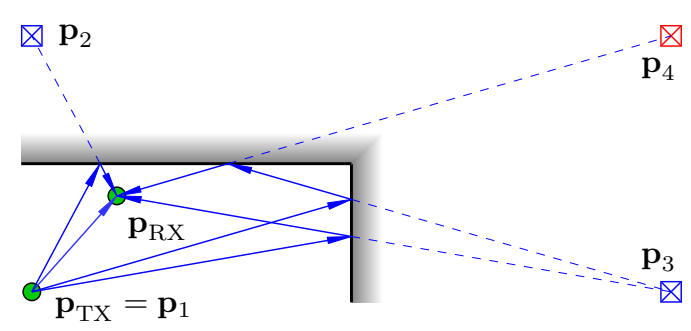

(a) Bistatic VAs

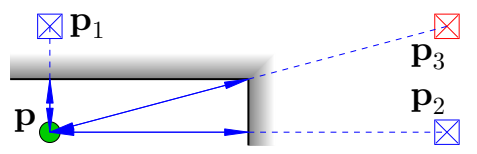

(b) Monostatic VAs

Fig. 1. Virtual anchors (VAs) on an exemplary indoor wall corner for bistatic and monostatic measurements. For (a), the LOS path is considered as VA $\mathbf{p}_{1}$ at $\mathbf{p}_{\mathrm{TX}}$. For $(\mathrm{b}), \mathbf{p}=\mathbf{p}_{\mathrm{RX}}=\mathbf{p}_{\mathrm{TX}}$ holds.
In the bistatic case, one agent acts as anchor and the cooperating partner agent is localized. An agent in monostatic mode acts as both transmitter and receiver, and the received signal represents an image of the surrounding geometry. ${ }^{2}$ The joint Cramér-Rao lower bound (CRLB) on the position error was analyzed in [3] for the cooperative approach, where monostatic contributions arise as additive Fisher information, valuable for self-localization [7], [8] for tracking agents over time. A related cooperative SLAM approach was studied in [9].

This paper presents an in-depth analysis of the position-related information provided by monostatic measurements, using a general form of multipath delay gradients and the CRLB on the position error. Monostatic measurements alone are unsuitable for robust agent localization. They do provide valuable ranging information to surrounding walls but geometric ambiguities are hard to resolve. The analysis of the monostatic CRLB reveals the position-related information content of such measurements, but also their limitations. Our key contributions are:

1) Derivation of the spatial multipath delay gradient, which connects geometry with the channel parameters. The general result covers all bistatic and monostatic setups.

2) Investigation of the CRLB on the monostatic position error using the previously derived gradients.

The paper is structured as follows. Section II introduces the used indoor UWB signal model and the relationship between position error CRLB and multipath delay gradients. Section III rigorously derives said gradient as a function of room geometry and node position. Section IV evaluates monostatic VA types and gives numerical results of the monostatic CRLB over an example room. Section V concludes the paper.

Notation: All vectors are column vectors and written boldface. Jacobians $\partial \boldsymbol{y} / \partial \boldsymbol{x}$ have dimension $\operatorname{dim} \boldsymbol{y} \times \operatorname{dim} \boldsymbol{x}$ but gradients $\partial s / \partial \boldsymbol{x}$ are column vectors $\operatorname{dim} \boldsymbol{x} \times 1$. $\mathbf{e}(\phi)=$ $[\cos (\phi), \sin (\phi)]^{\mathrm{T}}$ is a unit vector of tilt angle $\phi$.

\section{PROBlem Formulation}

\section{A. Signal Model}

We suppose a transmitted UWB pulse $s(t)$ of duration $T_{s}$ and employ for the received signal the model [1], [3]

$$
r(t)=\sum_{k=1}^{K} \alpha_{k} s\left(t-\tau_{k}\right)+(\nu * s)(t)+w(t)
$$

Gregor Dumphart thanks the co-authors for supervising his master's thesis at the Signal Processing and Speech Communication Laboratory of Graz University of Technology, which resulted in the research at hand.

${ }^{1}$ The terms bistatic and monostatic are well-established in radar.

${ }^{2}$ Think of a bat navigating in a cave: It emits ultrasonic sound, listens to the echoes and in this way estimates its position w.r.t. the nearby cave walls. 
over a total of $K$ considered paths. $\tau_{k} \in \mathbb{R}^{+}$are path delays, $\alpha_{k} \in \mathbb{C}$ complex path amplitudes and $k \in\{1 \ldots K\} . K$ is equivalent to the number of visible virtual anchors (VAs) at agent position p. Diffuse multipath (DM) is modeled as zero-mean Gaussian random process $\nu(t)$ with autocorrelation $R_{\nu}(\tau, u)=S_{\nu}(\tau) \delta(\tau-u)$, where we assume uncorrelated scattering. $w(t)$ is additive white Gaussian noise (AWGN) with two-sided power spectral density (PSD) $N_{0} / 2$.

Figure 1 illustrates the concept of multipath-assisted indoor localization: In Figure 1(a), a transmitter at position $\mathbf{p}_{\mathrm{TX}}$ isotropically sends an UWB pulse $s(t)$. The received signal includes, besides the line-of-sight path, reflections of the room walls, shown by the blue lines. These reflected paths can be modeled geometrically using VAs at positions $\mathbf{p}_{k}$, mirror images of the anchor w.r.t. walls that can be computed from the a priori known floor plan [10]. Since transmitter and receiver are separate, we call this the bistatic setup. For monostatic measurements, shown in Figure 1(b), the agent is both receiver and transmitter collocated at $\mathbf{p}$, and acts as anchor for itself with its own set of VAs at $\mathbf{p}_{k}$. In a cooperative multipath-assisted indoor localization setup [3], [6], both types of measurements are being used in order to perform robust, unambiguous position estimation.

The distance between agent $\mathbf{p}_{\mathrm{Rx}}$ and VA $\mathbf{p}_{k}$ equals the actual path length $c \tau_{k}$ [4], [11] where $c$ is the speed of light. Thus

$$
\tau_{k}=\frac{1}{c}\left\|\mathbf{p}_{\mathrm{Rx}}-\mathbf{p}_{k}\right\|_{2}
$$

constitutes the main link between geometry and signal model. We consider two-dimensional localization ${ }^{3}$, i.e. $\mathbf{p}_{\mathrm{Rx}}, \mathbf{p}_{k} \in \mathbb{R}^{2}$.

\section{B. CRLB on the Position Error}

We want to obtain an estimate $\hat{\mathbf{p}}$ of agent position $\mathbf{p}$ from the received signal $r(t)$ by exploiting the knowledge of the VA positions $\left\{\mathbf{p}_{k}\right\}$, in presence of DM and AWGN.

The position-related parameter vector $\boldsymbol{\theta}=\left[\mathbf{p}^{\mathrm{T}} \Re \boldsymbol{\alpha}^{\mathrm{T}} \Im \boldsymbol{\alpha}^{\mathrm{T}}\right]^{\mathrm{T}}$ where nuisance parameters $\Re \boldsymbol{\alpha}, \Im \boldsymbol{\alpha}$ are the real and imaginary parts of amplitudes $\boldsymbol{\alpha}=\left[\alpha_{1} \ldots \alpha_{K}\right]^{\mathrm{T}}$. $\mathcal{I}_{\boldsymbol{\theta}}$ is the Fisher information matrix (FIM) of $\boldsymbol{\theta}$. As described in [1], [3], [11], only the upper left block of its inverse $\left[\mathcal{I}_{\boldsymbol{\theta}}^{-1}\right]_{2 \times 2}=: \mathcal{I}_{\mathbf{p}}^{-1}$ is relevant to position estimation. $\mathcal{I}_{\mathrm{p}}$ is called equivalent Fisher information matrix and yields the CRLB on the position error

$$
\mathbb{E}_{\mathbf{r} \mid \boldsymbol{\theta}}\left\{(\hat{\mathbf{p}}-\mathbf{p})(\hat{\mathbf{p}}-\mathbf{p})^{\mathrm{T}}\right\} \geq \mathcal{I}_{\mathbf{p}}^{-1}
$$

The position error bound (PEB) is then the root of the trace

$$
\mathcal{P}(\mathbf{p})=\sqrt{\operatorname{tr}\left(\mathcal{I}_{\mathbf{p}}^{-1}\right)}
$$

$\mathcal{I}_{\boldsymbol{\theta}}$ is connected to signal model (1) by the chain rule

$$
\mathcal{I}_{\boldsymbol{\theta}}=\frac{\partial \boldsymbol{\psi}^{\mathrm{T}}}{\partial \boldsymbol{\theta}} \mathcal{I}_{\boldsymbol{\psi}} \frac{\partial \boldsymbol{\psi}}{\partial \boldsymbol{\theta}}
$$

where matrix $\mathcal{I}_{\psi}$ is the FIM of the channel parameter vector $\boldsymbol{\psi}=\left[\boldsymbol{\tau}^{\mathrm{T}}, \Re \boldsymbol{\alpha}^{\mathrm{T}}, \Im \boldsymbol{\alpha}^{\mathrm{T}}\right]^{\mathrm{T}}$ with $\boldsymbol{\tau}=\left[\tau_{1} \ldots \tau_{K}\right]^{\mathrm{T}}$ holding the delays. $\mathcal{I}_{\boldsymbol{\psi}}$ is given by likelihood function $f(\mathbf{r} \mid \boldsymbol{\psi})$ through

$$
\mathcal{I}_{\boldsymbol{\psi}}=\mathbb{E}_{\mathbf{r} \mid \boldsymbol{\psi}}\left\{\left(\frac{\partial \ln f(\mathbf{r} \mid \boldsymbol{\psi})}{\partial \boldsymbol{\psi}}\right)\left(\frac{\partial \ln f(\mathbf{r} \mid \boldsymbol{\psi})}{\partial \boldsymbol{\psi}}\right)^{\mathrm{T}}\right\}
$$

To obtain a more insightful expression for $\mathcal{I}_{\mathrm{p}}$, we break down the submatrices of Jacobian (4) and channel FIM $\mathcal{I}_{\psi}$ (5) to

$$
\frac{\partial \boldsymbol{\psi}}{\partial \boldsymbol{\theta}}=\left[\begin{array}{ll}
\partial \boldsymbol{\tau} / \partial \mathbf{p} & \mathbf{0}_{K \times 2 K} \\
\mathbf{0}_{2 K \times 2} & \boldsymbol{I}_{2 K \times 2 K}
\end{array}\right], \quad \boldsymbol{I}_{\boldsymbol{\psi}}=\left[\begin{array}{cc}
\boldsymbol{\Lambda}_{\mathrm{A}} & \boldsymbol{\Lambda}_{\mathrm{B}} \\
\boldsymbol{\Lambda}_{\mathrm{B}}^{\mathrm{T}} & \boldsymbol{\Lambda}_{\mathrm{C}}
\end{array}\right]
$$

where $\boldsymbol{\Lambda}_{\mathrm{A}} \in \mathbb{R}^{K \times K}, \boldsymbol{\Lambda}_{\mathrm{B}} \in \mathbb{R}^{K \times 2 K}, \boldsymbol{\Lambda}_{\mathrm{C}} \in \mathbb{R}^{2 K \times 2 K}$ are specified in Appendix C. By expanding the products of (4) using (6) and employing blockwise inversion lemma [3], [11], we obtain

$$
\mathcal{I}_{\mathbf{p}}=\frac{\partial \boldsymbol{\tau}^{\mathrm{T}}}{\partial \mathbf{p}}\left(\boldsymbol{\Lambda}_{\mathrm{A}}-\boldsymbol{\Lambda}_{\mathrm{B}} \boldsymbol{\Lambda}_{\mathrm{C}}^{-1} \boldsymbol{\Lambda}_{\mathrm{B}}^{\mathrm{T}}\right) \frac{\partial \boldsymbol{\tau}}{\partial \mathbf{p}} .
$$

The term in brackets describes the delay information of MPCs $\alpha_{k} s\left(t-\tau_{k}\right)$ in the noisy $r(t)$. The subtractive term represents the impact of path overlap which is significant whenever there is a pair of overlapping MPCs, i.e. $k, k^{\prime}$ that fulfill $\left|\tau_{k}-\tau_{k^{\prime}}\right|<$ $T_{s}$, but essentially zero otherwise [12].

The Jacobian $\partial \boldsymbol{\tau} / \partial \mathbf{p}$ translates delay information into position information. The underlying gradients $\partial \tau_{k} / \partial \mathbf{p}$ are determined by the surrounding room geometry at the current agent position. They show very particular behavior in the monostatic case, which is the focus of this paper.

For further analysis on the impact of the gradients on localization accuracy, we simplify (7) for non-overlapping MPCs. Then, $\boldsymbol{\Lambda}_{\mathrm{B}}$ is zero and $\boldsymbol{\Lambda}_{\mathrm{A}}$ diagonal, which by [11, eq. (15)] allows for decomposing $\mathcal{I}_{\mathrm{p}}$ into a sum over MPCs

$$
\mathcal{I}_{\mathbf{p}}=8 \pi^{2} \beta^{2} \sum_{k=1}^{K} \operatorname{SINR}_{k} \frac{\partial \tau_{k}}{\partial \mathbf{p}} \cdot \frac{\partial \tau_{k}}{\partial \mathbf{p}}
$$

where $\operatorname{SINR}_{k}=\left|\alpha_{k}\right|^{2} /\left(N_{0}+T_{s} S_{\nu}\left(\tau_{k}\right)\right)$ is the signal-tointerference-plus-noise ratio [3] and $\beta$ the effective bandwidth.

\section{Spatial Delay GRADIEnTS}

\section{A. Gradient Derivation in General Form}

As per (2), the length of the $k$-th path is

$$
c \tau_{k}=\left\|\mathbf{p}_{\mathrm{RX}}-\mathbf{p}_{k}\right\|=\sqrt{\left(x_{\mathrm{RX}}-x_{k}\right)^{2}+\left(y_{\mathrm{RX}}-y_{k}\right)^{2}}
$$

and the gradient w.r.t. some position $\mathbf{p} \in\left\{\mathbf{p}_{\mathrm{RX}}, \mathbf{p}_{\mathrm{TX}}\right\}$ is ${ }^{4}$

$$
\begin{aligned}
& c \frac{\partial \tau_{k}}{\partial \mathbf{p}}=\frac{x_{\mathrm{RX}}-x_{k}}{\sqrt{\left(x_{\mathrm{RX}}-x_{k}\right)^{2}+\left(y_{\mathrm{RX}}-y_{k}\right)^{2}}} \frac{\partial\left(x_{\mathrm{RX}}-x_{k}\right)}{\partial \mathbf{p}} \\
& \frac{y_{\mathrm{RX}}-y_{k}}{\sqrt{\left(x_{\mathrm{RX}}-x_{k}\right)^{2}+\left(y_{\mathrm{RX}}-y_{k}\right)^{2}}} \frac{\partial\left(y_{\mathrm{RX}}-y_{k}\right)}{\partial \mathbf{p}} \\
& =\cos \left(\phi_{k}\right)\left(\frac{\partial x_{\mathrm{RX}}}{\partial \mathbf{p}}-\frac{\partial x_{k}}{\partial \mathbf{p}}\right)+\sin \left(\phi_{k}\right)\left(\frac{\partial y_{\mathrm{RX}}}{\partial \mathbf{p}}-\frac{\partial y_{k}}{\partial \mathbf{p}}\right) \\
& =\left(\frac{\partial \mathbf{p}_{\mathrm{RX}}}{\partial \mathbf{p}}-\frac{\partial \mathbf{p}_{k}}{\partial \mathbf{p}}\right)^{\mathrm{T}} \cdot \mathbf{e}\left(\phi_{k}\right)
\end{aligned}
$$

${ }^{3}$ We refrain from a general 3D treatment because, to our current knowledge, results and especially derivation steps involving rotation matrices turn out considerably more complicated than in the 2D case. Fortunately, a 2D treatment is often sufficient for typical right-angled indoor environments.

${ }^{4}$ We refer to nodes as RX and TX by communications engineering habit, even though the particular roles and the signal flow direction is irrelevant in this context. This reciprocity is later emphasized by (22) and Figure 3. 
where $\mathbf{e}\left(\phi_{k}\right)=\left(\mathbf{p}_{\mathrm{RX}}-\mathbf{p}_{k}\right) /\left\|\mathbf{p}_{\mathrm{RX}}-\mathbf{p}_{k}\right\|$. Jacobian $\partial \mathbf{p}_{\mathrm{RX}} / \partial \mathbf{p}$ is identity if $\mathbf{p}=\mathbf{p}_{\mathrm{RX}}$ and zero otherwise. VAs $\mathbf{p}_{k}$ are a function of $\mathbf{p}_{\mathrm{TX}}$ and room geometry, thus $\partial \mathbf{p}_{k} / \partial \mathbf{p}$ is nonzero only for $\mathbf{p}=\mathbf{p}_{\mathrm{TX}}$. This motivates the use of indicator functions:

$$
\begin{gathered}
\mathbb{1}_{\mathrm{RX}}(\mathbf{p}):= \begin{cases}1 & \text { If } \mathbf{p}=\mathbf{p}_{\mathrm{RX}} \\
0 & \text { If } \mathbf{p} \neq \mathbf{p}_{\mathrm{RX}}\end{cases} \\
c \frac{\partial \tau_{k}}{\partial \mathbf{p}}=\left(\mathbb{1}_{\mathrm{RX}}(\mathbf{p}) \cdot \mathbf{I}_{2 \times 2}-\mathbb{1}_{\mathrm{TX}}(\mathbf{p}) \cdot \frac{\partial \mathbf{p}_{k}}{\partial \mathbf{p}_{\mathrm{TX}}}\right)^{\mathrm{T}} \cdot \mathbf{e}\left(\phi_{k}\right)
\end{gathered}
$$

Jacobian $\partial \mathbf{p}_{k} / \partial \mathbf{p}_{\mathrm{TX}}$, the last missing piece, is comprehensively derived in the following section.

\section{B. Derivation of the Virtual Anchor Jacobian}

We derive Jacobian $\partial \mathbf{p}_{k} / \partial \mathbf{p}_{\mathrm{TX}}$ in closed form as a function of node position and indoor geometry. Thereby, we examine the characteristics of the underlying reflections. We consider a fixed MPC, so w.l.o.g. we drop index $k$ and write $\partial \mathbf{p}_{\mathrm{VA}} / \partial \mathbf{p}_{\mathrm{TX}}$. Derivation and notation are guided by Figure 2.

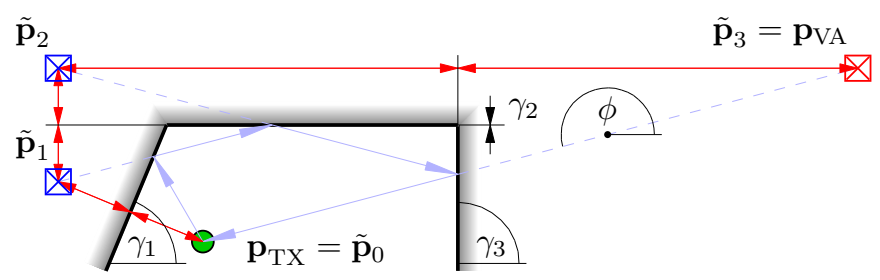

Fig. 2. Exemplary construction of a virtual anchor $\mathbf{p}_{\mathrm{VA}}$ via intermediate anchors $\widetilde{\mathbf{p}}_{q}$ by $Q$-fold mirroring of $\mathbf{p}_{\mathrm{Tx}}$ on the walls in such order as defined by the MPC at hand. Here, $Q=3, \gamma_{1}=3 \pi / 8, \gamma_{2}=0, \gamma_{3}=\pi / 2$.

We introduce index $q \in\{1, \ldots, Q\}$ to iterate the VA construction where $Q$ is the VA order (the number of reflections of the MPC at hand). Each $q$-step involves a particular wall, which we model with a line equation

$$
\mathbf{p}^{\prime}=t \cdot \mathbf{e}\left(\gamma_{q}\right)+\mathbf{d}_{q}
$$

where $t \in \mathbb{R}, \gamma_{q} \in(-\pi,+\pi]$ is the angle of the $q$-th wall, ${ }^{5}$ and $\mathbf{d}_{q} \in \mathbb{R}^{2}$ is a wall offset vector. In order to obtain the Jacobian, we have to find a representation $\mathbf{p}_{\mathrm{VA}}=$ $f\left(\mathbf{p}_{\mathrm{TX}}, \gamma_{1}, \mathbf{d}_{1}, \ldots, \gamma_{Q}, \mathbf{d}_{Q}\right)$ first. $\mathbf{p}_{\mathrm{VA}}$ is obtained by mirroring $\mathbf{p}_{\mathrm{TX}}$ on walls $Q$ times. We refer to intermediate positions as $\widetilde{\mathbf{p}}_{q}$ with the understanding that $\widetilde{\mathbf{p}}_{0}=\mathbf{p}_{\mathrm{TX}}$ and $\widetilde{\mathbf{p}}_{Q}=\mathbf{p}_{\mathrm{VA}}$. We obtain the $q$-th intermediate anchor position by mirroring position $q-1$ on the $q$-th wall, or more formally

$$
\widetilde{\mathbf{p}}_{q}=\mathcal{S}\left(\widetilde{\mathbf{p}}_{q-1}, \gamma_{q}, \mathbf{d}_{q}\right)
$$

where function $\mathcal{S}$ denotes mirroring. Considering $\mathbf{p}_{\mathrm{VA}}=\widetilde{\mathbf{p}}_{Q}$ and by using recursive substitution down to $q=0$, we get

$$
\mathbf{p}_{\mathrm{VA}}=\mathcal{S}\left(\ldots \mathcal{S}\left(\mathcal{S}\left(\mathbf{p}_{\mathrm{TX}}, \gamma_{1}, \mathbf{d}_{1}\right), \gamma_{2}, \mathbf{d}_{2}\right) \ldots, \gamma_{Q}, \mathbf{d}_{Q}\right) .
$$

Appendix A concretizes the mirroring function

$$
\begin{aligned}
\mathcal{S}\left(\widetilde{\mathbf{p}}_{q-1}, \gamma_{q}, \mathbf{d}_{q}\right) & =\mathbf{M}\left(\gamma_{q}\right) \cdot\left(\widetilde{\mathbf{p}}_{q-1}-\mathbf{d}_{q}\right)+\mathbf{d}_{q} \\
& =\mathbf{M}\left(\gamma_{q}\right) \cdot \widetilde{\mathbf{p}}_{q-1}+\left(\mathbf{I}-\mathbf{M}\left(\gamma_{q}\right)\right) \cdot \mathbf{d}_{q}
\end{aligned}
$$

where we use mirror matrix $\mathbf{M}\left(\gamma_{q}\right)$ that acts w.r.t. a line through the origin with angle $\gamma_{q}$,

$$
\begin{aligned}
\mathbf{M}\left(\gamma_{q}\right) & :=\left[\begin{array}{rr}
\cos \left(2 \gamma_{q}\right) & \sin \left(2 \gamma_{q}\right) \\
\sin \left(2 \gamma_{q}\right) & -\cos \left(2 \gamma_{q}\right)
\end{array}\right] \\
& =\mathbf{R}\left(2 \gamma_{q}\right) \cdot\left[\begin{array}{rr}
1 & 0 \\
0 & -1
\end{array}\right]=\mathbf{R}\left(2 \gamma_{q}\right) \cdot \mathbf{F} .
\end{aligned}
$$

Thus, mirroring $\mathbf{M}\left(\gamma_{q}\right)$ is the composition of sign-flip $\mathbf{F}$ in the second dimension

$$
\mathbf{F}=\left[\begin{array}{rr}
1 & 0 \\
0 & -1
\end{array}\right]
$$

and rotation $\mathbf{R}$ about angle $2 \gamma_{q}{ }^{6,7}$ For breaking down (11), we prefer (13) with the separated $\widetilde{\mathbf{p}}_{q-1}$-summand. Repeated application, where non-commutative matrices require care, yields an involved closed-form formula

$$
\begin{aligned}
\mathbf{p}_{\mathrm{VA}}=\left(\prod_{q=0}^{Q-1} \mathbf{M}\left(\gamma_{Q-q}\right)\right) \mathbf{p}_{\mathrm{TX}}+ \\
\quad \sum_{q=1}^{Q}\left(\prod_{\tilde{q}=1}^{Q-q} \mathbf{M}\left(\gamma_{Q+1-\tilde{q}}\right)\right)\left(\mathbf{I}-\mathbf{M}\left(\gamma_{q}\right)\right) \mathbf{d}_{q} .
\end{aligned}
$$

Fortunately, the derivative w.r.t. $\mathbf{p}_{\mathrm{TX}}$ is just the leading product

$$
\frac{\partial \mathbf{p}_{\mathrm{VA}}}{\partial \mathbf{p}_{\mathrm{TX}}}=\prod_{q=0}^{Q-1} \mathbf{M}\left(\gamma_{Q-q}\right)
$$

Transposition and symmetry $\mathbf{M}\left(\gamma_{q}\right)^{\mathrm{T}}=\mathbf{M}\left(\gamma_{q}\right)$ give

$$
\left(\frac{\partial \mathbf{p}_{\mathrm{VA}}}{\partial \mathbf{p}_{\mathrm{TX}}}\right)^{\mathrm{T}}=\prod_{q=1}^{Q} \mathbf{M}\left(\gamma_{q}\right) .
$$

To resolve this product, we use a pseudo-homomorphism property of the mirror matrix derived in Appendix B,

$$
\mathbf{M}(\gamma) \mathbf{M}(\beta)=\mathbf{M}(\gamma-\beta) \cdot \mathbf{F}
$$

By $(Q-1)$-fold application of (17) in (16), we obtain

$$
\begin{aligned}
\left(\frac{\partial \mathbf{p}_{\mathrm{VA}}}{\partial \mathbf{p}_{\mathrm{TX}}}\right)^{\mathrm{T}} & =\mathbf{M}\left(\gamma_{1}-\gamma_{2}+\gamma_{3}-\ldots+(-1)^{Q-1} \gamma_{Q}\right) \cdot \mathbf{F}^{Q-1} \\
& =\mathbf{M}(\bar{\gamma}) \cdot \mathbf{F}^{Q-1}=\mathbf{R}(2 \bar{\gamma}) \cdot \mathbf{F}^{Q}
\end{aligned}
$$

where (14) was used. We define the effective wall angle of the VA or MPC at hand

$$
\bar{\gamma}_{k}=\sum_{q=0}^{Q_{k}}(-1)^{q-1} \gamma_{k, q},
$$

an oscillated sum of the wall angles $\gamma_{k, q}$ involved in the reflection ${ }^{8}$ of the $k$-th path.

\footnotetext{
${ }^{5}$ In this context, we do not consider wall boundaries $t \in[a, b]$.

${ }^{6}$ Mirroring is hence also termed rotoreflection or improper rotation [13]

${ }^{7} \mathbf{M}\left(\gamma_{q}\right)$ has eigenvalue 1 along $\mathbf{e}\left(\gamma_{q}\right)$, i.e. in parallel to the wall, and -1 perpendicular. This, with $(12)$, formally reveals key properties of $\mathcal{S}$ - Invariance of parallel moves $\mathcal{S}(\mathbf{p}+t \cdot \mathbf{e}(\gamma), \gamma, \mathbf{d})=\mathcal{S}(\mathbf{p}, \gamma, \mathbf{d})+t \cdot \mathbf{e}(\gamma)$.

- Mirroring $\mathcal{S}(\mathbf{p}+t \cdot \mathbf{e}(\gamma+\pi / 2), \gamma, \mathbf{d})=\mathcal{S}(\mathbf{p}, \gamma, \mathbf{d})-t \cdot \mathbf{e}(\gamma+\pi / 2)$.

- Arbitrary choice of offset $\mathcal{S}(\mathbf{p}, \gamma, \mathbf{d}+t \cdot \mathbf{e}(\gamma))=\mathcal{S}(\mathbf{p}, \gamma, \mathbf{d})$.

${ }^{8}$ For a bistatic LOS path, $Q_{k}=0$ and (19) is an empty sum, thus $\bar{\gamma}_{k}=0$.

This makes our formalism compatible with conventional anchor utilization.
} 


\section{Gradient Summary and Conclusions}

Using (18) in (10) and the geometric meanings of $\mathbf{F}$ and $\mathbf{R}\left(2 \bar{\gamma}_{k}\right)$ leads to the final result for the spatial delay gradient:

$$
\begin{aligned}
c \frac{\partial \tau_{k}}{\partial \mathbf{p}} & =\left(\mathbb{1}_{\mathrm{RX}}(\mathbf{p}) \cdot \mathbf{I}-\mathbb{1}_{\mathrm{TX}}(\mathbf{p}) \cdot \mathbf{R}\left(2 \bar{\gamma}_{k}\right) \cdot\left[\begin{array}{cc}
1 & 0 \\
0 & (-1)^{Q_{k}}
\end{array}\right]\right) \cdot \mathbf{e}\left(\phi_{k}\right) \\
& =\mathbb{1}_{\mathrm{RX}}(\mathbf{p}) \cdot \mathbf{e}\left(\phi_{k}\right)-\mathbb{1}_{\mathrm{TX}}(\mathbf{p}) \cdot \mathbf{R}\left(2 \bar{\gamma}_{k}\right) \cdot \mathbf{e}\left((-1)^{Q_{k}} \phi_{k}\right) \\
& =\mathbb{1}_{\mathrm{RX}}(\mathbf{p}) \cdot \mathbf{e}\left(\phi_{k}\right)-\mathbb{1}_{\mathrm{TX}}(\mathbf{p}) \cdot \mathbf{e}\left((-1)^{Q_{k}} \phi_{k}+2 \bar{\gamma}_{k}\right)
\end{aligned}
$$

where $\bar{\gamma}_{k}$ is the effective wall angle (19) and $\phi_{k}$ is the angle of arrival of the $k$-th path. Indicator functions were defined in (9) and suggest the following case analysis.

For bistatic cases $\mathbf{p}_{\mathrm{RX}} \neq \mathbf{p}_{\mathrm{TX}}$, only one summand of (20) remains for a fixed choice $\mathbf{p} \in\left\{\mathbf{p}_{\mathrm{RX}}, \mathbf{p}_{\mathrm{TX}}\right\}$. Particularly,

$$
\frac{\partial \tau_{k}}{\partial \mathbf{p}_{\mathrm{RX}}}=\frac{1}{c} \mathbf{e}\left(\phi_{k}\right), \quad \frac{\partial \tau_{k}}{\partial \mathbf{p}_{\mathrm{TX}}}=-\frac{1}{c} \mathbf{e}\left((-1)^{Q_{k}} \phi_{k}+2 \bar{\gamma}_{k}\right) .
$$

Bistatic gradients w.r.t. TX can be simplified with the trivial property of forward and reverse path lengths being equal, i.e. $\tau_{k}=\tau_{k}^{\mathrm{rev}}$ and so $\left\|\mathbf{p}_{\mathrm{Rx}}-\mathbf{p}_{k}\right\|=\left\|\mathbf{p}_{\mathrm{Tx}}-\mathbf{p}_{k}^{\mathrm{rev}}\right\| .{ }^{9}$ The concept is shown in Figure 3. Considering switched roles for $\mathbf{p}_{\mathrm{RX}}$ and $\mathbf{p}_{\mathrm{TX}}$ then suggests

$$
\frac{\partial \tau_{k}}{\partial \mathbf{p}_{\mathrm{TX}}}=\frac{\partial \tau_{k}^{\mathrm{rev}}}{\partial \mathbf{p}_{\mathrm{TX}}}=\frac{1}{c} \mathbf{e}\left(\phi_{k}^{\mathrm{rev}}\right) .
$$

Then, (22) in (21) gives the (from a geometrical optics perspective) intuitive angle relation $\phi_{k}^{\text {rev }}=(-1)^{Q_{k}} \phi_{k}+2 \bar{\gamma}_{k}+\pi$.

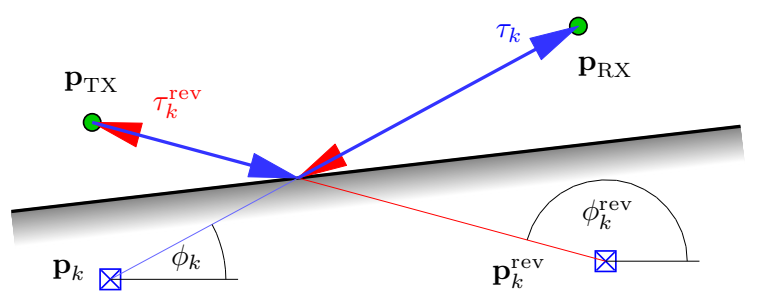

Fig. 3. Forward and reverse paths of a bistatic MPC and the corresponding VAs and angles of arrival.

In the monostatic case, we are facing a single mobile $\mathbf{p}=$ $\mathbf{p}_{\mathrm{TX}}=\mathbf{p}_{\mathrm{RX}}$ and so both summands of (20) remain.

$$
\begin{aligned}
\frac{\partial \tau_{k}}{\partial \mathbf{p}} & =\frac{1}{c}\left(\mathbf{e}\left(\phi_{k}\right)+\mathbf{e}\left(\phi_{k}^{\mathrm{rev}}\right)\right) \\
& = \begin{cases}\frac{2}{c} \sin \left(\bar{\gamma}_{k}\right) \mathbf{e}\left(\phi_{k}+\bar{\gamma}_{k}-\frac{\pi}{2}\right) & \text { If } Q_{k} \text { is even } \\
\frac{2}{c} \sin \left(\bar{\gamma}_{k}-\phi_{k}\right) \mathbf{e}\left(\bar{\gamma}_{k}-\frac{\pi}{2}\right) & \text { If } Q_{k} \text { is odd }\end{cases}
\end{aligned}
$$

Formula (23) has magnitude-times-direction form which is often useful for assessing monostatic reflections.

\section{RESUlTS}

\section{A. Evaluation of Monostatic Virtual Anchor Types}

We write the general gradient as magnitude times direction

$$
\frac{\partial \tau_{k}}{\partial \mathbf{p}}=\frac{1}{c} a_{k} \mathbf{e}\left(\mu_{k}\right)
$$

where $a_{k}$ represents spatial sensitivity and $\mathbf{e}\left(\mu_{k}\right)$ is the direction of greatest change of $\tau_{k}$. Monostatic gradients (23) satisfy $0 \leq a_{k} \leq 2$ while bistatic gradients (21) are of constant magnitude $a_{k}=1$. The reason is the two summands in (20): They represent changes in path length due to movement of $\mathbf{p}_{\mathrm{Rx}}$ and $\mathbf{p}_{\mathrm{TX}}$, respectively. For bistatic gradients, only one influence is active at a time, and the characteristics of either are alike, cf. (22). Monostatic gradients are affected by both, so the two influences may add up or cancel, depending on the multipath geometry, and thus allow for zero or double sensitivity.

Knowing the spatial delay gradients, we use (8) to write FIM $\mathcal{I}_{\mathrm{p}}$ as a sum of contributions from the individual VAs

$$
\mathcal{I}_{\mathbf{p}}=\frac{8 \pi^{2} \beta^{2}}{c^{2}} \sum_{k=1}^{K} \operatorname{SINR}_{k} a_{k}^{2} \mathbf{e}\left(\mu_{k}\right) \cdot \mathbf{e}\left(\mu_{k}\right)^{T}
$$

where $\mathbf{e}\left(\mu_{k}\right) \cdot \mathbf{e}\left(\mu_{k}\right)^{T}$ is a ranging direction matrix ${ }^{10}$ [11]

The above sum reveals that each MPC contributes onedimensional position information in direction $\mathbf{e}\left(\mu_{k}\right)$, scaled by the individual SINR and the square of spatial sensitivity $a_{k}$ [3], [11]. For bistatic cases, a technical evaluation is facilitated by $a_{k}=1$ and was done in [1]. For monostatic cases, $a_{k}$ depends heavily on the multipath geometry, which necessitates further investigation.

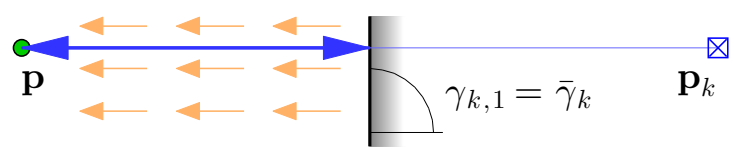

(a) $Q_{k}=1$ VA: $\bar{\gamma}_{k}=\gamma_{1}=\phi_{k} \pm \frac{\pi}{2}, a_{k}=2, \partial \tau_{k} / \partial \mathbf{p}=\frac{2}{c} \mathbf{e}\left(\phi_{k}\right)$.

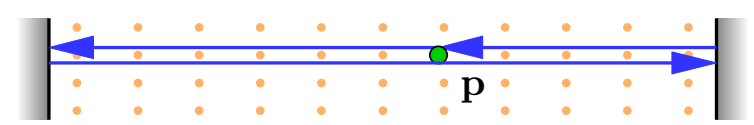

(b) $Q_{k}=2$ parallel walls VA: $\bar{\gamma}_{k}=0, a_{k}=0, \partial \tau_{k} / \partial \mathbf{p}=\mathbf{0}$.

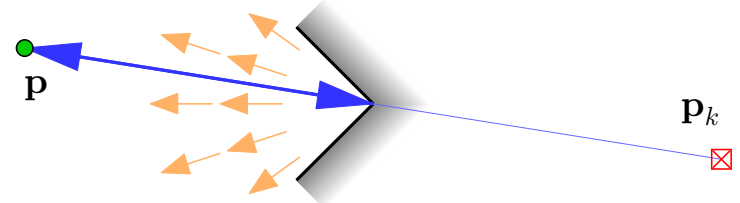

(c) $Q_{k}=2$ corner VA: $\bar{\gamma}_{k}= \pm \frac{\pi}{2}, a_{k}=2, \partial \tau_{k} / \partial \mathbf{p}=\frac{2}{c} \mathbf{e}\left(\phi_{k}\right)$.

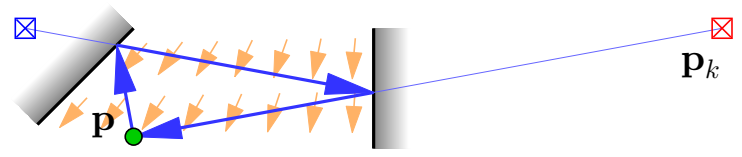

(d) $Q_{k}=2$ arbitrary VA: $\bar{\gamma}_{k}=\gamma_{1}-\gamma_{2}, a_{k}=2 \sin \left(\bar{\gamma}_{k}\right)$, $\partial \tau_{k} / \partial \mathbf{p}=\frac{2}{c} \sin \left(\bar{\gamma}_{k}\right) \mathbf{e}\left(\phi_{k}+\bar{\gamma}_{k}-\frac{\pi}{2}\right)$, cf. (23) even case.

Fig. 4. Monostatic VAs of order 1 and 2 and their properties for significant indoor geometries. The orange vector field illustrates the spatial delay gradient $\partial \tau_{k} / \partial \mathbf{p}$ and thus the direction of position information provided by the VA.

Figure 4 shows four important types of monostatic VAs in indoor setups. Figure 4(a) shows a first-order VA (i.e. a single reflection), providing position information orthogonal to the

${ }^{9} \tau_{k}^{\text {rev }}, \mathbf{p}_{k}^{\text {rev }}$ are just temporary symbols used to show the reciprocity idea. ${ }^{10} \mathbf{e}\left(\mu_{k}\right) \cdot \mathbf{e}\left(\mu_{k}\right)^{T}$ has eigenvalue 1 towards $\mathbf{e}\left(\mu_{k}\right)$ and 0 perpendicular. 
wall with twice the sensitivity $a_{k}$ than in bistatic cases (e.g., with anchors). Second-order reflections between parallel walls are useless for localization because of their constant delay: Figure 4(b) shows that the path length is twice the room width, independent of agent position. ${ }^{11,12}$ The gradient of the rectangular corner VA from Figure 4(c) is radially pointing away from the corner and has double sensitivity. For an arbitrary second-order VA like in Figure 4(d), the behavior is a mixture of the parallel and corner cases, depending on the wall angle mismatch.

VAs of order $Q_{k} \geq 3$ are insignificant in practice [10] since their SINR is usually below detectability, due to limited reflectivity of construction materials and path loss. Also, the PEB (3) is dominated by the few highest-SINR paths [12], so even detectable paths of $Q_{k} \geq 3$ would contribute very little.

\section{B. Numerical Results for the Monostatic PEB}

We assume a raised-cosine pulse of duration $T_{s}=1 \mathrm{~ns}$ on an $f_{c}=7 \mathrm{GHz}$ carrier. Like in [3], we set the power delay profile $S_{\nu}(\tau)$ of DM to a double-exponential (time constants: $5 \mathrm{~ns}$ rise, $20 \mathrm{~ns}$ decay). We use AWGN with double-sided PSD $N_{0} / 2$ such that $E_{\mathrm{LOS}} / N_{0}=80 \mathrm{~dB}$ at $1 \mathrm{~m}$ of propagation.

Figure 5 shows the floorplan of an example room, where the CRLB on the monostatic position error (3) is shown for agent positions therein. First-order and second-order corner VAs with $-3 \mathrm{~dB} \cdot Q_{k}$ attenuation are considered. The possible localization performance is particularly promising for agent positions close to the room corners. This is because an accurate position estimate requires high-SINR ranging information (i.e., low path loss and DM interference) in both horizontal and vertical directions, which is best provided by the nearest first-order VAs. Path overlap (PO) inevitably causes poor performance in certain regions: overlap of nearby first-order VAs leads to a performance drop along the room's symmetry axes. The parabola-shaped outage regions close to walls are due to a corner VA and an adjoining first-order VA having low delay separation there.

Figure 6 shows the upper left $3 \mathrm{~m} \times 3 \mathrm{~m}$ corner of the previous room for different assumptions. We observe how the additional consideration of second-order VAs helps performance in principle, but also leads to severe problems with PO.

For quantitative analysis, Figure 7 shows the CDF of PEB over the entire floor plan for pulse duration $T_{\mathrm{S}}=1 \mathrm{~ns}$ and different maximum VA orders (1 and 2). For the neglected PO case, one can observe that the PEB decreases when adding higher-order VAs (dashed lines). Obviously, PO leads to a degradation of the PEB, since useful position information is lost. This effect is even stronger when $Q_{k} \leq 2$, which is mostly due to overlap of first-order and corner paths close to walls, as described earlier.

\footnotetext{
${ }^{11}$ This property could prove useful in indoor mapping, i.e. estimation of an unknown floor plan from reflections. Observing those MPCs whose delays are invariant under agent movements could extend approaches like [14].

${ }^{12}$ The reverse path has the same constant length, causing inevitable overlap and singular $\Lambda_{C}^{\prime}, \mathcal{I}_{\mathrm{p}}$ when both VAs are considered. This problem affects all higher-order paths with non-coinciding directions of departure and arrival.
}

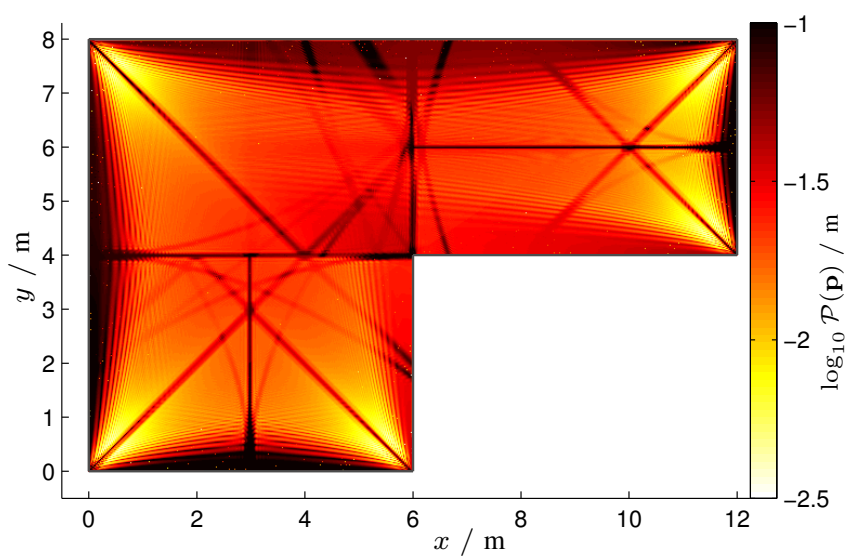

Fig. 5. Position error bound (PEB) of monostatic localization over a simple L-room. VAs are of order $Q_{k} \leq 2$ and path overlap (PO) is considered.

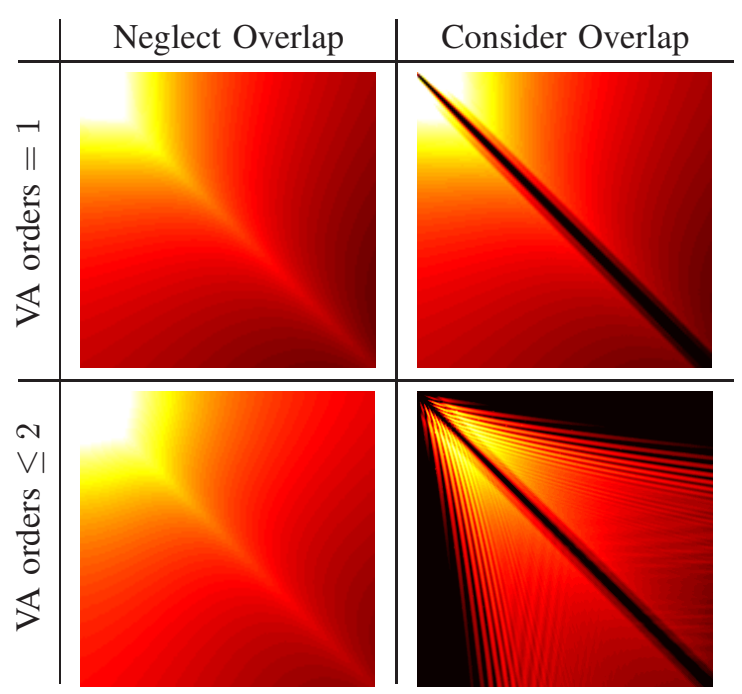

Fig. 6. The upper left $3 \mathrm{~m} \times 3 \mathrm{~m}$ corner of the room in Figure 5 for different parameters. Here, the value range $[$ white, black $] \hat{=}\left[10^{-2.5} \mathrm{~m}, 10^{-1.5} \mathrm{~m}\right]$.

\section{CONCLusions}

The CRLB on the position error of monostatic multipathassisted indoor localization has been decomposed into Jacobians of multipath delays over agent position and the Fisher information matrix of signal model parameters. To compute the Jacobians, we formulated the spatial gradients of multipath delays. This general result shows that the influence of room geometry reduces to a single scalar quantity, termed effective wall angle. We analyzed the gradient for the most significant types of monostatic VAs to understand their behavior. The latter could be used for measuring room dimensions.

Numerical results show that, besides outage regions caused by path overlap, monostatic concepts are applicable for highly accurate node localization. However, monostatic position estimation is intrinsically ambiguous and utilizing it for robust localization is possible only as part of a more sophisticated concept, e.g. a cooperative approach. 


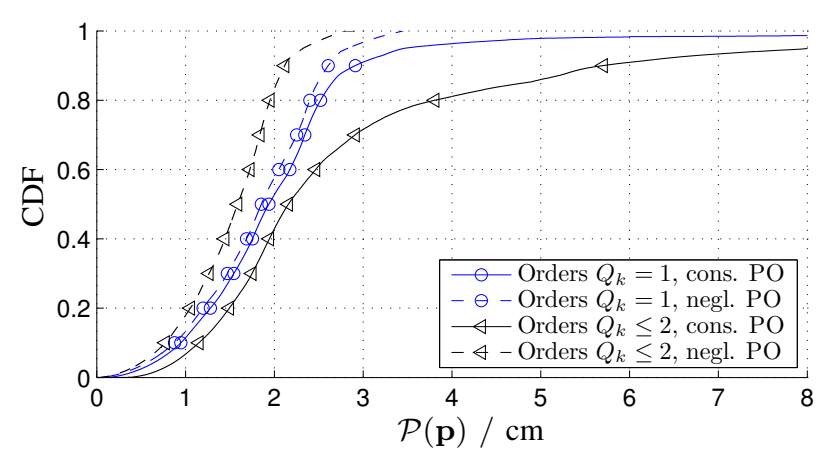

Fig. 7. CDFs of the PEB with $T_{\mathrm{S}}=1 \mathrm{~ns}$ for VAs of order one and two. Path overlap is neglected in the results marked by dashed lines.

\section{APPENDIX A}

ProOF OF MirRoring Formula (12)

Recall definition (15) of $\mathbf{F}$ and (14) of $\mathbf{M}(\gamma)$. To find a formula for mirror image $\mathcal{S}(\mathbf{p}, \gamma, \mathbf{d})$ of $\mathbf{p}$ about wall $\gamma, \mathbf{d}$, we first switch to a more suitable coordinate system where wall and horizontal axis coincide. To do so, we move the origin onto the wall by subtracting $\mathbf{d}$ and then compensate the wall angle with rotation $\mathbf{R}(-\gamma)$, so $\mathbf{p}$ moves to $\mathbf{p}^{\prime}=\mathbf{R}(-\gamma)(\mathbf{p}-\mathbf{d})$. Now, mirroring equals a simple vertical flip $\mathbf{p}_{\text {mir }}^{\prime}=\mathbf{F} \mathbf{p}^{\prime}$. Lastly, we undo coordinate transformations $\mathbf{p}_{\text {mir }}=\mathbf{R}(\gamma) \mathbf{p}_{\text {mir }}^{\prime}+\mathbf{d}$. Broken down to $\mathbf{p}$, the mirror image is located at

$$
\mathcal{S}(\mathbf{p}, \gamma, \mathbf{d})=\mathbf{p}_{\text {mir }}=\mathbf{R}(\gamma) \mathbf{F R}(-\gamma)(\mathbf{p}-\mathbf{d})+\mathbf{d} .
$$

Some basic trigonometry reveals $\mathbf{R}(\gamma) \mathbf{F R}(-\gamma)=\mathbf{M}(\gamma)$, thus

$$
\mathcal{S}(\mathbf{p}, \gamma, \mathbf{d})=\mathbf{M}(\gamma)(\mathbf{p}-\mathbf{d})+\mathbf{d}
$$

\section{APPENDIX B}

\section{Proof of Mirror MATrix Property (17)}

Mirror matrices $\mathbf{M}(\gamma)$ are symmetric and orthogonal and thus self-inverse. Sign-flip $\mathbf{F}=\mathbf{M}(0)$ inherits these properties. Rotation is a well-known homomorphism $\mathbf{R}(\gamma) \mathbf{R}(\beta)=\mathbf{R}(\gamma+\beta)$. Definition $\mathbf{M}(\gamma)=\mathbf{R}(2 \gamma) \mathbf{F}$ from (14) implies $\mathbf{R}(2 \gamma)=$ $\mathbf{M}(\gamma) \mathbf{F}$. We rearrange the following product to derive the desired pseudo-homomorphism property of the mirror matrix:

$$
\begin{gathered}
\begin{aligned}
\mathbf{M}(\gamma) \mathbf{M}(\beta)= & \mathbf{M}(\gamma) \mathbf{M}(\beta)^{\mathrm{T}}=\mathbf{R}(2 \gamma) \mathbf{F}(\mathbf{R}(2 \beta) \mathbf{F})^{\mathrm{T}} \\
= & \mathbf{R}(2 \gamma) \mathbf{F F}^{-1} \mathbf{R}(2 \beta)^{\mathrm{T}}=\mathbf{R}(2 \gamma) \mathbf{R}(-2 \beta) \\
= & \mathbf{R}(2(\gamma-\beta))=\mathbf{M}(\gamma-\beta) \cdot \mathbf{F}
\end{aligned} \\
\text { APPENDIX C } \\
\text { LiKeliHOOD FUNCTION AND FISHER INFORMATION } \\
\text { MATRIX OF SIGNAL MODEL PARAMETERS }
\end{gathered}
$$

\section{APPENDIX C}

\section{LIKELIHOOD FUNCTION AND FISHER INFORMATION}

The likelihood function (LHF) of the sampled received signal $\mathbf{r}$ in AWGN and diffuse multipath (DM) is given as [1], [3]

$$
f(\mathbf{r} \mid \psi) \propto \exp \left\{2 \Re\left\{\mathbf{r}^{\mathrm{H}} \mathbf{C}_{\mathrm{n}}^{-1} \mathbf{S} \boldsymbol{\alpha}\right\}-\boldsymbol{\alpha}^{\mathrm{H}} \mathbf{S}^{\mathrm{H}} \mathbf{C}_{\mathrm{n}}^{-1} \mathbf{S} \boldsymbol{\alpha}\right\}
$$

where $\mathbf{S}=\left[\mathbf{s}_{\tau_{1}}, \ldots, \mathbf{s}_{\tau_{K}}\right] \in \mathbb{R}^{N \times K}$ is the signal matrix containing delayed versions $\mathbf{s}_{\tau_{k}}=\left[s\left(T_{\mathrm{s}}-\tau_{k}\right), \ldots, s\left(N T_{\mathrm{s}}-\tau_{k}\right)\right]^{\mathrm{T}}$ of the sampled transmit pulse and $\mathbf{C}_{\mathrm{n}}=\sigma_{\mathrm{n}}^{2} \mathbf{I}_{N \times N}+\mathbf{C}_{\mathrm{c}}$ is the noise co-variance matrix. AWGN samples have variance $\sigma_{\mathrm{n}}^{2}=$ $N_{0} / T_{\mathrm{s}}$; the elements of the DM co-variance matrix are given by $\left[\mathbf{C}_{\mathrm{c}}\right]_{n, m}=T_{\mathrm{s}} \sum_{i=1}^{N} S_{\nu}\left(i T_{\mathrm{s}}\right) s\left(n T_{\mathrm{s}}-i T_{\mathrm{s}}\right) s\left(m T_{\mathrm{s}}-i T_{\mathrm{s}}\right)$. Using (C.1) in (5) yields FIM $\mathcal{I}_{\psi}$ (see (6)), whose submatrices are given [3] as follows:

$$
\begin{gathered}
\boldsymbol{\Lambda}_{\mathrm{B}}=\left[\begin{array}{ll}
\boldsymbol{\Lambda}_{\mathrm{B}}^{R} & \boldsymbol{\Lambda}_{\mathrm{B}}^{I}
\end{array}\right], \quad \boldsymbol{\Lambda}_{\mathrm{C}}=\left[\begin{array}{ll}
\boldsymbol{\Lambda}_{\mathrm{C}}^{\prime} & 0 \\
0 & \boldsymbol{\Lambda}_{\mathrm{C}}^{\prime}
\end{array}\right] \\
{\left[\boldsymbol{\Lambda}_{\mathrm{A}}\right]_{k, k^{\prime}}=2 \Re\left(\alpha_{k} \alpha_{k^{\prime}}^{*}\left(\frac{\partial \mathbf{s}_{\tau_{k^{\prime}}}}{\partial \tau_{k^{\prime}}}\right)^{\mathrm{H}} \mathbf{C}_{\mathbf{n}}^{-1} \frac{\partial \mathbf{s}_{\tau_{k}}}{\partial \tau_{k}}\right)} \\
{\left[\boldsymbol{\Lambda}_{\mathrm{B}}^{R}\right]_{k, k^{\prime}}=2 \Re\left(\alpha_{k}\left(\mathbf{s}_{\tau_{k^{\prime}}}\right)^{\mathrm{H}} \mathbf{C}_{\mathbf{n}}^{-1} \frac{\partial \mathbf{s}_{\tau_{k}}}{\partial \tau_{k}}\right)} \\
{\left[\boldsymbol{\Lambda}_{\mathrm{B}}^{I}\right]_{k, k^{\prime}}=2 \Im\left(\alpha_{k}\left(\mathbf{s}_{\tau_{k^{\prime}}}\right)^{\mathrm{H}} \mathbf{C}_{\mathbf{n}}^{-1} \frac{\partial \mathbf{s}_{\tau_{k}}}{\partial \tau_{k}}\right)} \\
{\left[\boldsymbol{\Lambda}_{\mathrm{C}}^{\prime}\right]_{k, k^{\prime}}=2 \Re\left(\left(\mathbf{s}_{\tau_{k}}\right)^{\mathrm{H}} \mathbf{C}_{\mathbf{n}}^{-1} \mathbf{s}_{\tau_{k^{\prime}}}\right)} \\
\operatorname{REFERENCES}
\end{gathered}
$$

\section{REFERENCES}

[1] K. Witrisal and P. Meissner, "Performance bounds for multipath-assisted indoor navigation and tracking (MINT)," in Communications (ICC), 2012 IEEE International Conference on, June 2012, pp. 4321-4325.

[2] P. Meissner, E. Leitinger, and K. Witrisal, "UWB for Robust Indoor Tracking: Weighting of Multipath Components for Efficient Estimation," Wireless Communications Letters, IEEE, vol. 3, no. 5, pp. 501-504, Oct 2014.

[3] E. Leitinger, P. Meissner, C. Ruedisser, G. Dumphart, and K. Witrisal, "Evaluation of Position-related Information in Multipath Components for Indoor Positioning," IEEE Journal on Selected Areas in Communications, 2015, to appear.

[4] J. Borish, "Extension of the image model to arbitrary polyhedra," The Journal of the Acoustical Society of America, vol. 75, no. 6, 1984.

[5] N. Michelusi, U. Mitra, A. Molisch, and M. Zorzi, "UWB Sparse/Diffuse Channels, Part I: Channel Models and Bayesian Estimators," IEEE Transactions on Signal Processing, vol. 60, no. 10, pp. 5307-5319, Oct 2012.

[6] M. Froehle, E. Leitinger, P. Meissner, and K. Witrisal, "Cooperative Multipath-Assisted Indoor Navigation and Tracking (Co-MINT) Using UWB Signals," in IEEE ICC 2013 Workshop on Advances in Network Localization and Navigation (ANLN), June 2013, pp. 16-21.

[7] Y. Shen, H. Wymeersch, and M. Win, "Fundamental Limits of Wideband Localization - Part II: Cooperative Networks," IEEE Transactions on Information Theory, vol. 56, no. 10, pp. 4981-5000, Oct 2010.

[8] Y. Shen, S. Mazuelas, and M. Win, "Network Navigation: Theory and Interpretation," IEEE Journal on Selected Areas in Communications, vol. 30, no. 9, pp. 1823-1834, October 2012.

[9] M. des Noes and B. Denis, "Benefits from cooperation in simultaneous anchor-less tracking and room mapping based on Impulse Radio - Ultra Wideband devices," in International Conference on Systems, Signals and Image Processing (IWSSIP), April 2012, pp. 17-21.

[10] P. Meissner and K. Witrisal, "Analysis of Position-Related Information in Measured UWB Indoor Channels," in 6th European Conference on Antennas and Propagation (EuCAP), March 2012, pp. 6-10.

[11] Y. Shen and M. Win, "Fundamental Limits of Wideband Localization Part I: A General Framework," Information Theory, IEEE Transactions on, vol. 56, no. 10, pp. 4956-4980, Oct 2010.

[12] — , "Effect of Path-Overlap on Localization Accuracy in Dense Multipath Environments," in Communications, 2008. ICC '08. IEEE International Conference on, May 2008, pp. 4197-4202.

[13] H. Coxeter, Introduction to Geometry, ser. Wiley Classics Library. Wiley, 1989.

[14] T. Deissler and J. Thielecke, "UWB SLAM with Rao-Blackwellized Monte Carlo data association," in Indoor Positioning and Indoor Navigation (IPIN), 2010 International Conference on, Sept 2010. 